\title{
Frequency, Extent, and Correlates of Superficial Siderosis and Ependymal Siderosis in Premature Infants with Germinal Matrix Hemorrhage: An SWI Study
}

\author{
(1) M.S. Albayram, (1) G. Smith, 다. Tufan, and (1DM.D. Weiss
}

\begin{abstract}
BACKGROUND AND PURPOSE: Germinal matrix intraventricular hemorrhage is a common complication of prematurity. An underrecognized complication of germinal matrix intraventricular hemorrhage is superficial siderosis, and the clinical consequences of superficial siderosis are not well-known. We aimed to investigate the prevalence, anatomic distribution, and severity of superficial siderosis and ependymal siderosis in premature infants with germinal matrix intraventricular hemorrhage using SWI.
\end{abstract}

MATERIALS AND METHODS: In this retrospective study, we included 88 patients across all grades of germinal matrix intraventricular hemorrhage who underwent MR imaging at term-equivalent age. Images were evaluated for the presence, distribution, and severity of superficial siderosis and ependymal siderosis. Univariate and multivariate logistic regression analyses were performed to determine factors associated with superficial siderosis and ependymal siderosis. The agreement among $\mathrm{T}$, $\mathrm{T} 2$, and $\mathrm{SWI}$ sequences was examined.

RESULTS: Seventy-two patients had brain stem superficial siderosis, and 79 patients had ependymal siderosis. The presence, extent, and severity of superficial siderosis and ependymal siderosis were closely related to the grade of germinal matrix intraventricular hemorrhage and intraventricular hematoma volume. Brain stem superficial siderosis had a stronger correlation with intraventricular hemorrhage than with cerebellar hemorrhage. Compared with SWI, T1 and T2 sequences detected only small proportions of patients with superficial siderosis $(12.5 \%$ and $6.9 \%$, respectively).

CONCLUSIONS: The incidence of superficial siderosis and ependymal siderosis is very high in preterm infants with germinal matrix intraventricular hemorrhage when assessed by SWI at term-equivalent age. The presence and extent of superficial siderosis and ependymal siderosis are closely related to germinal matrix intraventricular hemorrhage grade and intraventricular hematoma volume. Additional prospective studies using SWI are needed to clearly determine the clinical consequences of germinal matrix intraventricular hemorrhage with superficial siderosis and ependymal siderosis.

ABBREVIATIONS: $\mathrm{CN}=$ cranial nerve; $\mathrm{ES}=$ ependymal siderosis; GM-IVH = germinal matrix intraventricular hemorrhage; HUS = head ultrasound; SS = superficial siderosis; TVHV $=$ total ventricular hematoma volume

S uperficial siderosis (SS) of the CNS is a disease characterized by deposition of hemosiderin, a by-product of the breakdown of red blood cells, into subpial layers of CNS tissue caused by chronic or recurrent bleeding into the subarachnoid space. ${ }^{1,2}$ In adults, most cases of CNS SS present with sensorineural hearing loss and cerebellar ataxia. Myelopathy, polyradiculopathy, anosmia, and dementia may also be present. ${ }^{1,2}$ Despite the attention

Received September 23, 2019; accepted after revision November 12.

From the Departments of Radiology (M.S.A., G.S.) and Pediatrics (M.D.W.)

University of Florida College of Medicine, Gainesville, Florida; and Silivrikapi mh Hisaralti cd. Fatih sitesi Al/9 (F.T.), Fatih, Istanbul, Turkey.

Please address correspondence to Mehmet Sait Albayram, MD, Department of Radiology, University of Florida College of Medicine, PO Box 100374, Gainesville, FL 32610-0374; e-mail: albaym@radiology.ufl.edu

http://dx.doi.org/10.3174/ajnr.A6371

afforded to imaging findings and clinical sequelae of adult SS, few studies have described SS, ependymal siderosis (ES), or similar pathology occurring after germinal matrix intraventricular hemorrhage (GM-IVH) in premature infants. ${ }^{3-5}$ To the best of our knowledge, no previous study has investigated the relationship of GM-IVH to SS or ES using primarily SWI, the most sensitive MR imaging for the detection of hemorrhage and siderosis. ${ }^{6-8}$

This study aimed to investigate the prevalence and anatomic distribution of SS and ES in premature infants with GM-IVH of different severities using SWI to assess the relationship of GMIVH grade with the presence of SS in the cranial nerve (CN) VII/ VIII complex and to determine the factors primarily associated with brain stem SS. We hypothesized that GM-IVH grade and volume would be positively related to the severity of SS and ES. 


\section{MATERIALS AND METHODS Study Population}

Patients with GM-IVH who underwent MR imaging in our institution (Department of Radiology, University of Florida College of Medicine) between 2011 and 2018 were identified by a search in our electronic data base for the key words "germinal matrix hemorrhage." The study was approved by the institutional review board, and parental informed consent was obtained. Sex, birth weight, and gestational age at birth were noted. Inclusion criteria were as follows: 1) a history of GM-IVH diagnosed on head ultrasound (HUS) within 3 weeks after birth, and 2) the availability of at least $1 \mathrm{MR}$ imaging study of diagnostic quality including axial SWI, T1, and T2-weighted imaging at term-equivalent age. Patients were excluded from the study if the quality of imaging was insufficient to recognize the presence of SS, if additional clinical diagnoses such as meningitis or abscess were present, or if MR imaging was performed before term-equivalent age. Patients in whom GM-IVH was detected on HUS but not MR imaging were also excluded.

\section{Image Acquisition}

MR imaging was performed on a 1.5T scanner (Avanto; Siemens, Erlangen, Germany) or a 3T scanner (Verio; Siemens) between 2011 and 2018 with a multichannel head coil in our radiology department. At our institution, MR imaging in neonates is routinely performed without sedation or general anesthesia. MR imaging was typically performed at term-equivalent age before discharge. For each patient, axial SWI was performed. Magnitude images, SWI, and SWI-minimum-intensity-projection images were obtained automatically from the scanner.

\section{Image Analysis}

Analysis of all images was performed by M.S.A., a neuroradiology attending physician with extensive experience in neuroimaging research. GM-IVH grading and measurements of hematoma size were performed using the patients' HUS studies during the acute GM-IVH phase for the purpose of this study. The Papile classification was used for grading of GM-IVH with respect to the findings of the HUS examination in which hemorrhage was detected. ${ }^{9}$ GM-IVH and cerebellar hemorrhage dimensions (anterior-posterior [AP], craniocaudal [CC], and transverse diameter) were recorded in each hemisphere, and GM-IVH volume was calculated by the ellipsoid volume formula $\mathrm{AP} \times \mathrm{CC} \times$ transverse $\times \pi / 6$ for each hemisphere. ${ }^{10}$ Total dimensions of the ventricular hematoma included both germinal matrix and ventricular hemorrhage values combined. All calculations, visual evaluations, and measurements were performed on the PACS software (Visage; http://www.visageimaging.com). The presence of SS and ES was visually determined using magnitude, SWI, and minimum-intensity-projection SWI. Measurements of SS or ES depth were made using SWI.

The presence or absence of siderosis and maximal siderosis depth measurements were recorded from the bilateral lateral ventricles, bilateral lateral ventricular choroid plexuses, ependyma of the third ventricle, choroid plexus of the third ventricle, ependyma of the fourth ventricle, choroid plexus of the fourth ventricle, vermis of the cerebellum, bilateral cerebellar hemispheres, pons, mesencephalon, medulla oblongata, and spinal cord. The presence or absence of SS in the CN VII/VIII complex was also noted. Last, the distributions of SS in the spinal cord, medulla oblongata, pons, and mesencephalon were visually estimated as $0^{\circ}, 90^{\circ}, 180^{\circ}, 270^{\circ}$, or $360^{\circ}\left(0^{\circ}\right.$ denoting no SS and $360^{\circ}$ denoting SS around the entire perimeter of a structure).

\section{Statistics}

Statistical analysis was performed using SPSS 20.0 (IBM, Armonk, New York). The normality of distribution of continuous data was assessed with the Kolmogorov-Smirnov or ShapiroWilk tests. Categoric variables were presented as frequencies and percentages, and continuous variables were presented as mean $\pm \mathrm{SD}$ or median (minimum-maximum) when appropriate. Categoric variables were compared using $\chi^{2}$ and Fisher exact tests, and continuous variables were compared using Mann-Whitney $U$, Student $t$, ANOVA, or Kruskal-Wallis tests. The correlations between variables were assessed using Pearson or Spearman correlation tests. Binary logistic regression was performed to determine factors independently associated with the presence of SS in the brain stem. The variables associated with brain stem SS in univariate analyses were included in logistic regression models. Because of the strong correlation between GM-IVH grade and total ventricular hematoma volume (TVHV), 2 separate logistic-regression models were used, each including one of these factors. The level of agreement between MR imaging sequences in the detection of brain stem SS was analyzed using $\kappa$ analysis. Statistical significance was established at $P<.05$.

\section{RESULTS}

A total of 88 patients (52 males and 36 females) with GM-IVH were included. The mean gestational age at birth was $27.5 \pm$ 2.9 weeks. The mean birth weight was $1110.6 \pm 505.6 \mathrm{~g}$. The mean durations between birth and HUS and MR imaging examinations were $6.1 \pm 3.3$ days and $68.5 \pm 32.7$ days, respectively. There were 22 patients in each GM-IVH grade group. While 33 patients had cerebellar hematoma (median volume, $14.7 \mathrm{~mm}^{3}$; minimum-maximum volume, $0.5-6333 \mathrm{~mm}^{3}$ ), siderosis was present in the ependymal zone of 79 (Fig 1), brain stem of 72 (Fig 2 ), cerebellar vermis of 53 , cerebellum of 31 , and CNs VII and VIII of 42. All patients with brain stem SS and 6/15 of patients without brain stem SS had ES. All patients with cerebellar SS also had cerebellar vermis SS, and all patients with cerebellar vermis SS also had brain stem SS. Supratentorial SS was detectable in $34 \%$ (30/88), mainly in the Sylvian fissures bilaterally. Convexity SS was present only in 2 patients (2.2\%). Table 1 documents the relationship of GM-IVH grade to patient demographics, presence or absence of siderosis, and depth of siderosis in different anatomic locations.

Brain stem SS and ES were significantly more common in the GM-IVH grades II, III, and IV compared with the GM-IVH grade I group ( $P<.001$ for all comparisons). The depth of brain stem SS increased with higher GM-IVH grades (for grades I-III, $P<.001$ for all comparisons) and was similar between patients with grade III and grade IV GM-IVH. Brain stem SS distribution and ventricular ependymal SS depth also increased in parallel 

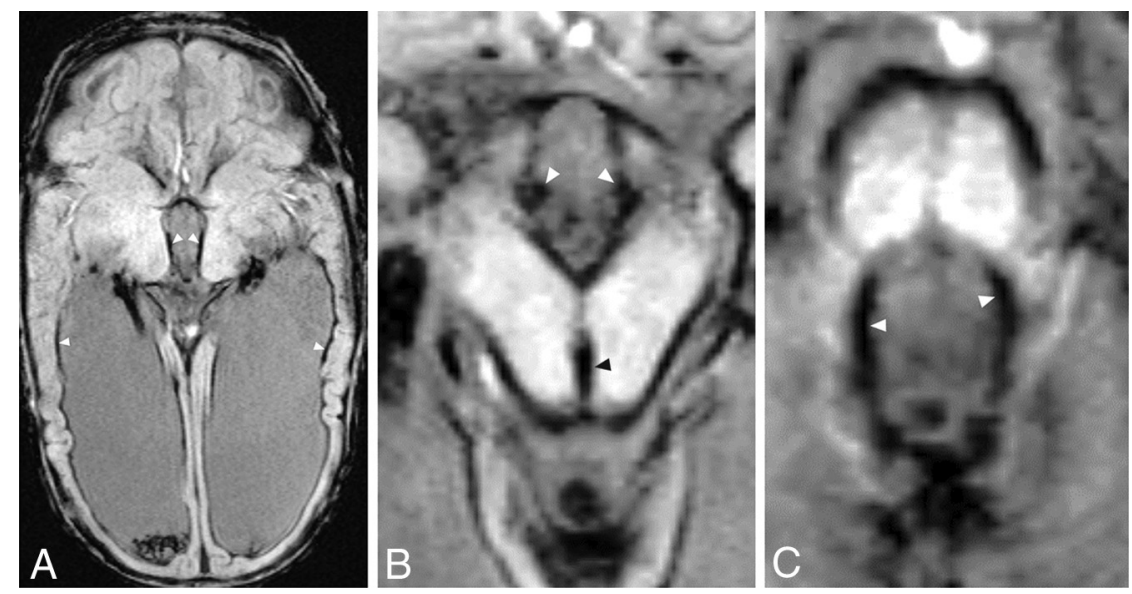

FIG 1. Axial SWI of a premature male infant born at 30 weeks' gestation diagnosed with grade III GM-IVH shows significant siderosis in the ependymal surfaces at the level of the lateral ventricles ( $A$, arrowheads), third ventricle ( $B$, white arrowheads), cerebral aqueduct ( $B$, black arrowhead), and fourth ventricle ( $C$, arrowheads). MR imaging was performed 14 weeks after birth. Note significant SS around the mesencephalon and pons. with the severity of GM-IVH grade $(P<.001$ for all comparisons, Table 1 and Fig 3). While the incidence of cerebellar SS tended to be greater in higher GM-IVH grades $(P=.009)$, the incidence of cerebellar hematoma was similar among GM-IVH grade groups. The median cerebellar SS depth in-creased in parallel with the severity of GM-IVH grade $(P=.001$, Table 1$)$. While the TVHV significantly increased in higher GM-IVH grade groups $(P<.001)$, there was no such trend for cerebellar hematoma volume $(P=.12)$.

In the present study, we detected a significant SWI signal decrease, suggestive of blood products, on the surface of the CN VII and VIII complex (Fig 4). This finding was seen in $47 \%$ of patients in the present study (42/88).
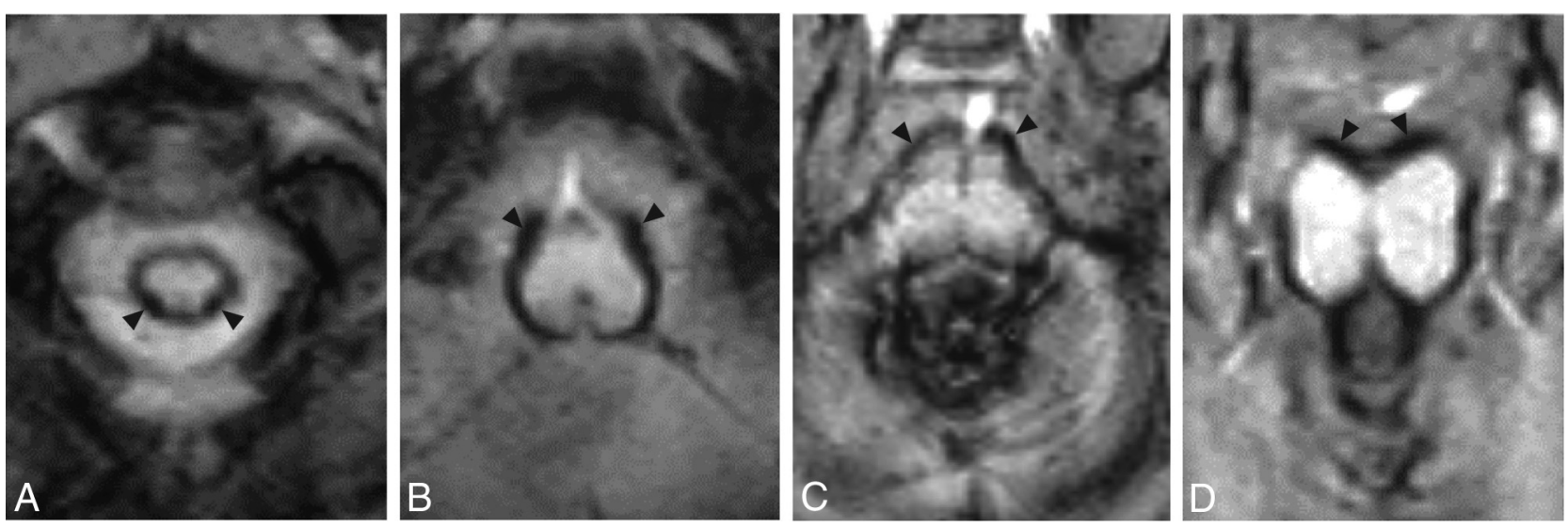

FIG 2. Axial SWI depicting SS in the same patient as in Fig 1 at the level of the cervical spinal cord $(A)$, medulla oblongata (B), pons (C), and mesencephalon (D). Arrowheads indicate the point of maximal SS depth within each structure. Note the circumferential distribution of SS around each of these brain stem structures and significant vermian SS.

Table 1: Comparison of GM-IVH groups for study variables ${ }^{\mathrm{a}}$

\begin{tabular}{|c|c|c|c|c|c|}
\hline GM-IVH Grade & I & II & III & IV & $P$ \\
\hline Brain stem SS (\%) & 45.5 & 95.5 & 95.5 & 95.5 & $<.001$ \\
\hline Brain stem SS distribution & $0(140.6)$ & $168.8(140.6)$ & $213.8(106.9)$ & $247.5(163.1)$ & $<.001$ \\
\hline Spinal cord SSD (mm) & $0(0-0.8)$ & $0.65(0-1.1)$ & $0.8(0-1.5)$ & $0.75(0-1.5)$ & $<.001$ \\
\hline Medulla oblongata SSD (mm) & $0(0-0.8)$ & $0.75(0-1.2)$ & $1(0-1.7)$ & $1(0-2)$ & $<.001$ \\
\hline Pons SSD (mm) & $0(0-1.2)$ & $0.6(0-1.1)$ & $0.8(0-1.7)$ & $0.85(0-1.8)$ & $<.001$ \\
\hline Mesencephalon SSD (mm) & $0(0-0.9)$ & $0.6(0-1.1)$ & $0.8(0-1.6)$ & $0.8(0-2.3)$ & $<.001$ \\
\hline Lateral ventricle SSD (mm) & $0.5(0-1)$ & $0.9(0-3.3)$ & $1(0-2)$ & $0.95(0.4-2.5)$ & $<.001$ \\
\hline Third ventricle SSD (mm) & $0(0-0.2)$ & $0(0-1.6)$ & $0(0-2.2)$ & $0.6(0-1.2)$ & $<.001$ \\
\hline Fourth ventricle SSD (mm) & $0(0-0.8)$ & $0.1(0-0.9)$ & $0.4(0-1.9)$ & $0.65(0-2.2)$ & $<.001$ \\
\hline CN VII/VIII siderosis (\%) & 9.1 & 54.5 & 72.7 & 54.5 & $<.001$ \\
\hline Total ventricular HV on HUS $\left(\mathrm{mm}^{3}\right)$ & $64.9(79.8)$ & $491.1(358.7)$ & $2168.7(2469)$ & $4319.4(9731.5)$ & $<.001$ \\
\hline Total cerebellar HV $\left(\mathrm{mm}^{3}\right)$ & $0(0-13.6)$ & $0(0-6333)$ & $0(0-530)$ & $0.5(0-1123)$ & .12 \\
\hline Cerebellar SS present (\%) & 31.8 & 77.3 & 72.7 & 59.1 & .009 \\
\hline Cerebellar SS depth (mm) & $0(0-0.25)$ & $0.3(0-2)$ & $0.35(0-2.1)$ & $0.43(0-2.2)$ & .001 \\
\hline Cerebellar hematoma present (\%) & 27.3 & 45.5 & 27.3 & 50 & .26 \\
\hline
\end{tabular}

Note:-HV indicates hematoma volume; SSD, superficial siderosis depth

${ }^{a}$ Numeric variables are presented as median (minimum-maximum). 

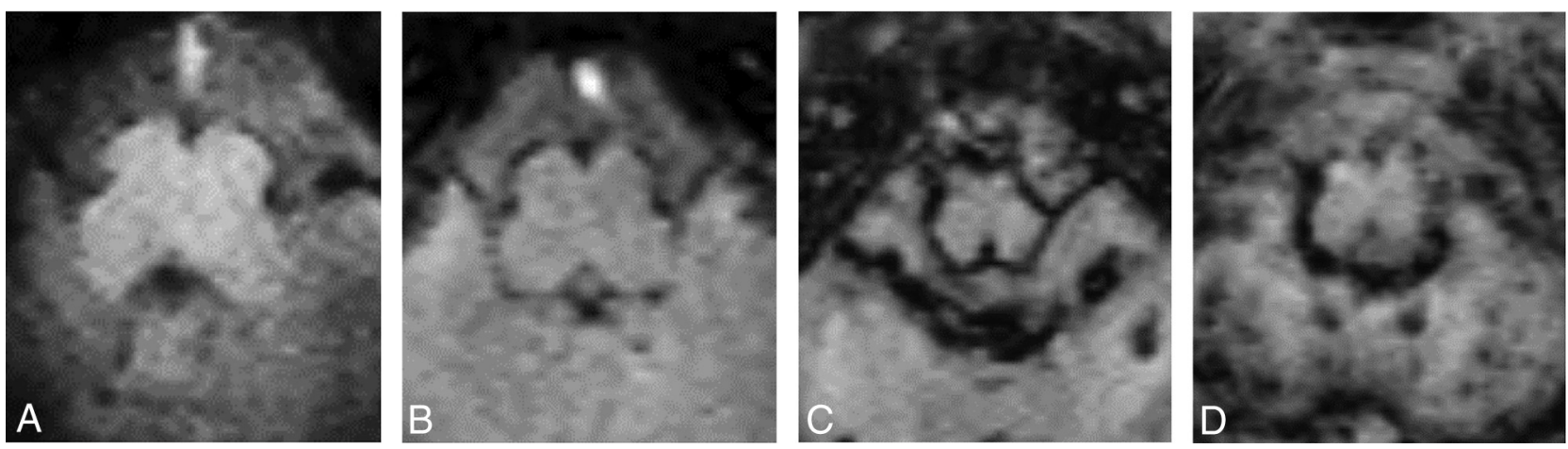

FIG 3. Sample axial SWI views at the level of the medulla oblongata in infants diagnosed with GM-IVH grades I (A), II (B), III (C), and IV (D). Note that severity of SS, measured by SS depth and distribution, increases from lower grade to higher grade GM-IVH. Also note the multiple punctate hemorrhages seen in the cerebellum in grades III and IV GM-IVH.
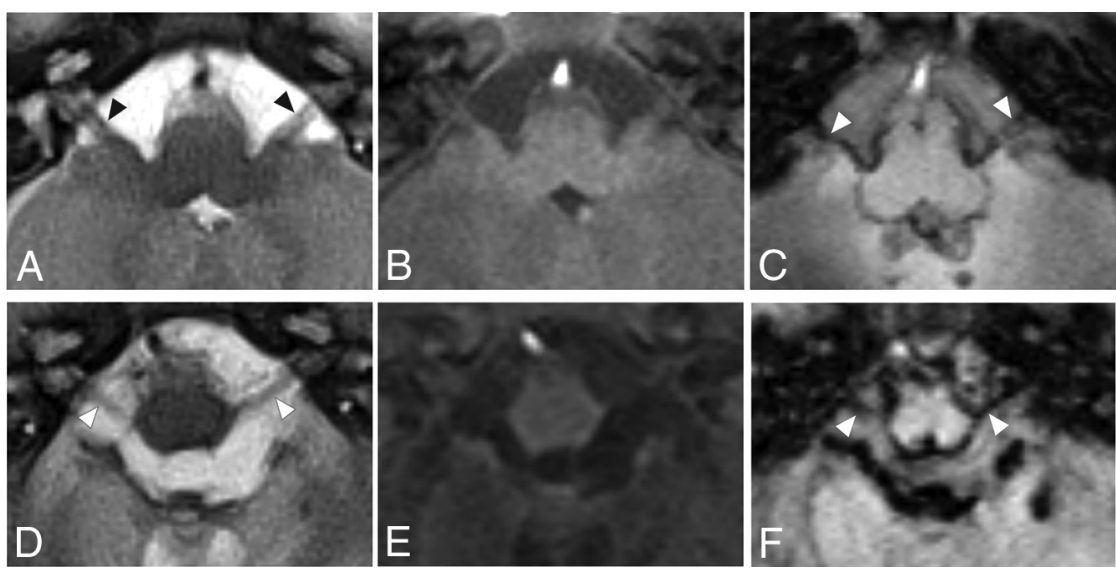

FIG 4. Axial MR images from 2 patients at the level of CNs VII and VIII seen on T2-weighted imaging $(A$ and $D)$, Tl-weighted imaging $(B$ and $E)$, and SWI ( $C$ and $F$ ). The arrowhead indicates the positions of the cisternal segment of CNs VII and VIII. Leptomeningeal thickening and significant iron accumulation can be seen in CNs VII and VIII bilaterally. Images were obtained from a boy born at 28 weeks' gestation with grade III GM-IVH $(A-C)$ and a boy born at 24 weeks' gestation with grade III GM-IVH $(D-F)$.

The incidence of CN VII and VIII SS increased in parallel with GM-IVH grades for grades I-III (linear-by-linear association $P<.001$ ), but the grade IV group had a rate equivalent to that of the grade II group (Table 1). The strongest correlations with the presence of CN VII and VIII SS were siderosis of the lateral ventricle $(r=0.62, P<.001)$ and choroid plexus of the fourth ventricle $(r=0.6, P<.001)$.

The GM-IVH grade and TVHV had moderate-to-strong positive correlations with brain stem ( $r$ range, 0.52-0.6; $P<.001$ for all), cerebellar ( $r=0.37, P<.001)$, and ventricular ES depth $(r$ range, $0.4-0.67 ; P<.001$ for all) (Table 2). Cerebellar hematoma volume had positive-but-weak correlations with brain stem SS depth measurements ( $r$ range, $0.2-0.34$; $P$ range, .001-.06) and a moderate and positive correlation with cerebellar SS depth $(r=$ $0.46, P<.001)$.

The patients with brain stem SS had a significantly higher GM-IVH grade $(P<.001)$, ventricular SS depth $(P<.001)$, incidence of CN VII and VIII SS $(P<.001)$, TVHV $(P<.001)$, cerebellar vermis SS depth $(P<.001)$, and cerebellar SS depth $(P=$ .002) (Table 3 ). The patients with and without brain stem SS had similar rates and volumes of cerebellar hematoma and cerebellar vermis hematoma $(P>.05$ for all comparisons).

The binary logistic regression analysis indicated that both GM-IVH grade (model $r^{2}=0.48$; OR $=5.9 ; 95 \%$ CI, 2.1-16.3; $P=.001)$ and total ventricle volume (model $r^{2}=0.36$; OR = 1.001; 95\% CI, 1-1.003; $P=.04$ ) had independent and significant associations with the presence of brain stem SS.

Among the patients with brain stem SS, the T1 sequence detected $9 / 72$ patients $(12.5 \%)$, while the T2 sequence detected $5 / 72$ patients (6.9\%). The level of agreement for the presence of brain stem SS between SWI and T1 sequences was $0.049(P=.15)$, and the level of agreement between SWI and T2 sequences was 0.026 $(P=.30)$.

\section{DISCUSSION}

Intracortical injection of ferrous or ferric chloride into the rat or cat cortex causes transient epileptiform discharge, focal edema, and cavitary gliosis. ${ }^{11,12}$ Repeat subarachnoid bleeding and incident circulation of heme in the CSF can result in SS, ${ }^{13}$ a rare disorder characterized by intra- and extracellular hemosiderin deposition into subpial tissues. ${ }^{14-16}$ Neuropathology studies show that SS particularly affects the ventricular ependyma, choroid plexus epithelium, inferior temporal lobes, cerebellum, brain stem, spinal cord, and CNs VII and VIII. ${ }^{14-16}$ Microscopically, the meninges may be thickened and fibrotic, ${ }^{16}$ and hemosiderinladen macrophages and extracellular iron granules can be identified in the subpial brain stem parenchyma, spinal cord, and cerebellum. ${ }^{14-16}$ Cases of brown-staining subpial siderosis and ES have been found extending approximately $2 \mathrm{~mm}$ in the spinal cord and 1-3 $\mathrm{mm}$ in the brain stem inward into the neural parenchyma. ${ }^{14,16}$ Central chromatolysis in the anterior horn cells and 
Table 2: Correlations between GM-IVH grades and hematoma volumes with $\mathrm{SS}^{\mathrm{a}}$

\begin{tabular}{|c|c|c|c|c|c|c|}
\hline & \multicolumn{2}{|c|}{$\begin{array}{c}\text { GM-IVH } \\
\text { Grade }\end{array}$} & \multicolumn{2}{|c|}{$\begin{array}{c}\text { Total } \\
\text { Ventricular } \\
\mathrm{HV}\left(\mathrm{mm}^{3}\right) \\
\end{array}$} & \multicolumn{2}{|c|}{$\begin{array}{l}\text { Cerebellar } \\
\mathrm{HV}\left(\mathrm{mm}^{3}\right)\end{array}$} \\
\hline & $r$ & $P$ & $r$ & $P$ & $r$ & $P$ \\
\hline Spinal cord SSD (mm) & 0.55 & $<.001$ & 0.55 & $<.001$ & 0.29 & .07 \\
\hline Medulla oblongata SSD (mm) & 0.60 & $<.001$ & 0.63 & $<.001$ & 0.30 & .005 \\
\hline Pons SSD (mm) & 0.52 & $<.001$ & 0.61 & $<.001$ & 0.20 & .06 \\
\hline Mesencephalon SSD (mm) & 0.59 & $<.001$ & 0.62 & $<.001$ & 0.34 & .001 \\
\hline Brain stem SS distribution & 0.54 & $<.001$ & 0.62 & $<.001$ & 0.25 & .018 \\
\hline Lateral ventricle SSD (mm) & 0.48 & $<.001$ & 0.51 & $<.001$ & 0.32 & .02 \\
\hline Third ventricle SSD (mm) & 0.46 & $<.001$ & 0.66 & $<.001$ & 0.28 & .009 \\
\hline Fourth ventricle SSD (mm) & 0.44 & $<.001$ & 0.59 & $<.001$ & 0.21 & .049 \\
\hline Choroid lateral ventricle SSD (mm) & 0.67 & $<.001$ & 0.72 & $<.001$ & 0.26 & .013 \\
\hline Choroid third ventricle SSD (mm) & 0.61 & $<.001$ & 0.73 & $<.001$ & 0.32 & .003 \\
\hline $\begin{array}{l}\text { Choroid fourth ventricle SSD } \\
(\mathrm{mm})\end{array}$ & 0.40 & $<.001$ & 0.47 & $<.001$ & 0.23 & .03 \\
\hline Cerebellar SSD (mm) & 0.37 & $<.001$ & 0.50 & $<.001$ & 0.46 & $<.001$ \\
\hline $\begin{array}{l}\text { Total ventricular HV on HUS } \\
\left(\mathrm{mm}^{3}\right)\end{array}$ & 0.85 & $<.001$ & $\mathrm{n} / \mathrm{a}$ & $\mathrm{n} / \mathrm{a}$ & 0.22 & .04 \\
\hline Cerebellar HV $\left(\mathrm{mm}^{3}\right)$ & 0.17 & .11 & 0.22 & .04 & $\mathrm{n} / \mathrm{a}$ & $\mathrm{n} / \mathrm{a}$ \\
\hline
\end{tabular}

Note:-n/a indicates not applicable; HV, hematoma volume; SSD, superficial siderosis depth; HUS, head ultrasound.

${ }^{a}$ GMH-IVH hemorrhage was detected by HUS and classified by using the Papile classification.

Table 3: Comparison of patients with and without brain stem SS

\begin{tabular}{lccc}
\hline & $\begin{array}{c}\text { Brain Stem SS } \\
\text { Absent }(\boldsymbol{n}=15)\end{array}$ & $\begin{array}{c}\text { Brain Stem SS } \\
\text { Present }(\boldsymbol{n}=73)\end{array}$ & $\boldsymbol{P}$ \\
\hline Gestational age at birth (wk) & $28.6 \pm 2.9$ & $27.3 \pm 2.9$ & .11 \\
Sex (\% male) & 53.3 & 60.3 & .62 \\
Birth weight (g) & $1299.5 \pm 830$ & $1071.7 \pm 407.4$ & .11 \\
GM-IVH grade & $1(0)$ & III (2) & $<.001$ \\
Total ventricular HV on HUS $\left(\mathrm{mm}^{3}\right)$ & $91.6(96.3)$ & $935.1(2978.8)$ & $<.001$ \\
Cerebellar hematoma $(\%)$ & 20 & 35.6 & .24 \\
Cerebellar HV (mm $\left.{ }^{3}\right)$ & $0(0)$ & $0(7.9)$ & .12 \\
CV hematoma + (\%) & 0 & 19.2 & .12 \\
CV HV (mm $\left.{ }^{3}\right)$ & $0(0)$ & $0(0)$ & .07 \\
Cerebellar SS + (\%) & 0 & 42.5 & .001 \\
Lateral ventricle SSD (mm) & $0(0-1)$ & $0.9(0-3.3)$ & $<.001$ \\
Third ventricle SSD (mm) & $0(0-0)$ & $0(0-2.2)$ & $<.001$ \\
Fourth ventricle SSD (mm) & $0(0-0)$ & $0.4(0-2.2)$ & $<.001$ \\
CV SS + (\%) & 0 & 72.6 & $<.001$ \\
CN VII/VIII SS + (\%) & 0 & 57.5 & $<.001$ \\
CV SS depth (mm) & $0(0-0)$ & $0.4(0-2.8)$ & $<.001$ \\
Cerebellar SS depth (mm) & $0(0-0)$ & $0(0-2.5)$ & .002 \\
\hline
\end{tabular}

Note:-CV indicates cerebellar vermis; GM-IVH, Germinal matrix intraventricular hemorrhage; HV, hematoma volume; SS, superficial siderosis; CN, cranial nerve; +, positive, pathology found; SSD, superficial siderosis depth.

${ }^{a}$ Numeric variables are presented as mean \pm SD or median (minimum-maximum) where appropriate.

system are sufficient to induce patterns of neuropathology similar to those seen in human SS within approximately 23 months.

Previous studies have documented neuropathologic findings of shortterm survivors of intraventricular hemorrhage. One study found that of 20 infants with verified intraventricular hemorrhage who survived for at least 1 week, 9 were found to have clotted blood filling dilated ventricles, suggesting severe GM-IVH and posthemorrhagic hydrocephalus. ${ }^{19}$ In all except 1 of these 9 cases, an accumulation of blood pigment was noted in the subarachnoid space. This study did not describe the location of subarachnoid blood products in the posterior fossa. Non-protein-bound iron has been found frequently and at high levels in CSF from preterm infants as early as 2-3 weeks after GM-IVH. ${ }^{20}$ In premature infants, GM-IVH may also precipitate $\mathrm{SS}^{3-5}$ SS secondary to neonatal intraventricular hemorrhage was first described by Gomori et al, ${ }^{4}$ who reported findings on T2-weighted imaging of superficial hypointensity of the medulla, tectal region, and Sylvian fissures in a neonate patient with bilateral GM-IVH. A later study identified low signal patterns on heavily T2WI consistent with SS in the basal cisterns, along the tentorium, covering the brain stem, and in the Sylvian fissures of 7 infants with previous peri-/ intraventricular hemorrhage. ${ }^{3}$ More recently, Yilmaz et $\mathrm{al}^{5}$ reported SS in a retrospective study of 37 patients with a history of GM-IVH. SS was detected using T2-weighted imaging or SWI in $67.6 \%(25 / 37)$ of patients, was present

ovoid/spheroid bodies (a marker distinctive of SS) may also be present between the myelinated axons in the brain stem and in the subpial white matter in the spinal cord. ${ }^{13,16}$ SS commonly presents with hearing impairment, ${ }^{1,2}$ which appears to be related to the vulnerability of CN VIII to SS.

Due to the limited number of human studies of SS and the indolent course of disease progression, studies of SS in animal models have been critical to establish disease pathophysiology and the timing required for pathologic features of the disease to emerge. Studies in which autologous washed blood cells were injected weekly during 3-6 months into the cisterna magna of rabbits identified distinct patterns of iron accumulation extending $1-2 \mathrm{~mm}$ in the brain parenchyma after 3 months. ${ }^{17,18}$ These animal studies indicate that bolus infusions of blood products into the ventricular infratentorially in all 25 patients with SS, with additional supratentorial SS in $27 \%$ (10/37). There was no discussion provided about the presence or distribution of SS in the posterior fossa, CNs, brain stem, cerebellum, or spinal cord or the relationship between the severity of GM-IVH and the severity of SS.

In the present study, $81 \%$ of preterm infants with GM-IVH showed some degree of SS in the posterior fossa, and $90 \%$ showed supratentorial ES. Supratentorial SS was detected in only $34 \%$ of cases, mainly in the Sylvian fissures and, rarely (2\%), in the cerebral convexities. The study by Yilmaz et $\mathrm{al}^{5}$ reported a frequency of ES higher than that of our study but did not include cases of grade I GM-IVH. Our study noted a higher frequency of posterior fossa SS than the study of Yilmaz et al, which we believe is most likely related to our exclusive 

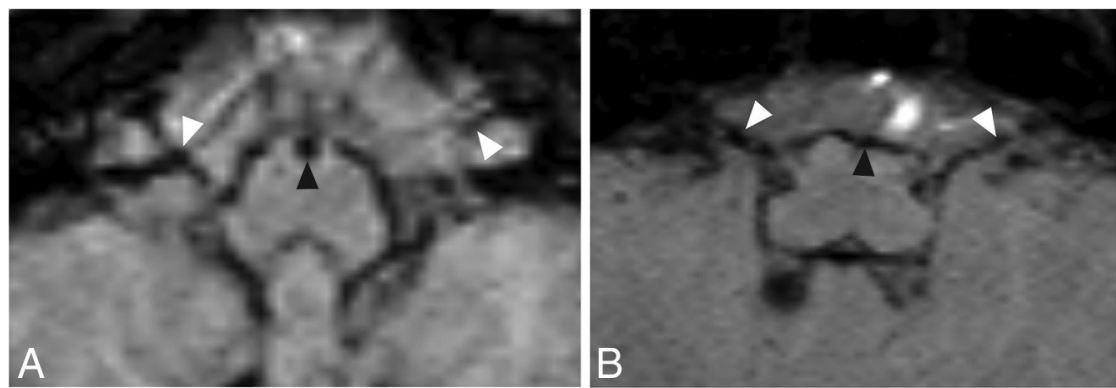

FIG 5. Initial axial SWI shows significant SS as early as 12 days after birth in a male patient with grade IV GM-IVH in the medulla oblongata ( $A$, black arrowhead) and the CN VII/VIII complex ( $A$, white arrowheads). Follow-up axial SWI of this patient reveals significant residual SS in the medulla oblongata (B, black arrowhead) and CN VII/VIII complex (B, white arrowheads) at 235 days after birth. Note that significant residual SS can also be seen in the CN VII/VIII complex at both time points.

use of SWI, which appears to be more sensitive to SS. We detected SS in the cerebellar vermis of $60 \%(53 / 88)$ of patients and in the cerebellar hemispheres of $35 \%$ (31/88). Hemispheric cerebellar SS in our population was less common than vermian SS. We believe this is related to the close proximity of the vermis to the mesencephalon and supravermian cistern. In the present study, SS in the posterior fossa was most frequent and severe within the brain stem. The depth of SS was greatest at the level of medulla oblongata, which is adjacent to both the foramen of Luschka and fourth ventricle outlets. SS was less severe in the mesencephalon. The timing at which MRIs were collected for the present study (mean, $68.5 \pm 32.7$ days) aligns with the timeframe during which we would expect iron accumulation and parenchymal destruction following GM-IVH, based on experimental studies as discussed earlier. It is not clear, however, how long SS remains detectable in infants after the initial hemorrhage. One case showed evidence of significant residual SS at 235 days after GM-IVH (Fig 5), suggesting that toxic effects of SS could be present, in some cases, for several months before resolution.

We found that both GM-IVH grade and TVHV were closely associated with SS depth in the brain stem, ventricle surfaces, cerebellum, and spinal cord. Multivariate regression analysis suggested that the presence of brain stem SS was associated with GM-IVH grade and TVHV, independent of birth weight, gestational age at birth, and cerebellar hematoma volume. Furthermore, cerebellar and vermis SS were present in many cases without cerebellar or vermis hemorrhage. This finding suggests that rather than cerebellar germinal matrix hemorrhage, intraventricular hemorrhage is the main factor underlying both brain stem and cerebellar SS.

In the present study, ES was common in both the supratentorial and infratentorial regions and was closely associated with GM-IVH grade, TVHV, and brain stem SS. Notably, all patients with brain stem SS and $40 \%$ of patients without brain stem SS had ES. These findings suggest that ES is a common consequence of GM-IVH. Chronic or subacute iron accumulation in the ependymal surfaces may have a deleterious effect on subsequent brain development, in addition to the effects of GMIVH itself, due to the close proximity of these structures to the germinal matrix. The germinal matrix, which is a key source of oligodendroglial precursor cells, produces myelin in the cerebral white matter. ${ }^{21,22}$ Infants with posthemorrhagic hydrocephalus have been found to exhibit ferritin-positive reactive microglia in the fourth ventricular wall before 2 weeks of age, in addition to hemosiderin deposits, nodular gliosis, ependymal cell loss, and subependymal rosettes in the fourth ventricular wall after 2 weeks of age. ${ }^{23}$ Further studies are needed to investigate the clinical significance of ES and possible damage to the germinal matrix.

CN VIII is especially vulnerable to iron accumulation in adult SS, corresponding to the clinical presentation of hearing loss in most adult patients with SS. ${ }^{1}$ In 1 postmortem case study of an adult with SS and sensorineural hearing loss, pathologic examination revealed severe bilateral degeneration of the organ of Corti and spiral ganglion cells, with iron deposits noted in the glial and other cells of the cochlear nucleus in the brain stem near its CSF-facing surface. ${ }^{24}$ There is concern that similar patterns of SS may contribute adversely to neurodevelopmental outcomes in premature infants. A recent cohort study of extremely preterm infants reported higher rates of hearing loss at 2- to 3-years' corrected age in infants with GM-IVH (I-IV included) compared with infants without GM-IVH. ${ }^{25}$ In the present study, we detected significant iron accumulation on the surfaces of the CN VII/VIII complex. This finding was seen in $47 \%$ of patients in the present study (42/88). The incidence increased from grade I to III, but the grade IV group had a rate equivalent to that of the grade II group. The features most strongly correlated with the presence of $\mathrm{CN}$ VII/VIII SS were lateral ventricle ES and fourth ventricle choroid plexus ES. This finding is likely related to the close proximity of CNs VII and VIII and the lateral fourth ventricle outlets, in which the fourth ventricle choroid plexuses are present bilaterally.

The SWI sequence is the MR imaging sequence most sensitive to hemorrhage and $S^{7}$ and was used in all patients of this study. Previous imaging studies of GM-IVH generally used the T1, T2, or gradient $\mathrm{T} 2$ sequence to detect SS. In the present study, T1 and T2 sequences detected only a small proportion of the patients with SS. These findings suggest that SWI is more sensitive and efficient for detecting the presence and severity of SS compared to $\mathrm{T} 1$ and $\mathrm{T} 2$ weighted imaging.

The present study is limited by its retrospective design, the use of 2 different MR imaging machines, and the absence of a second radiologist to determine interrater reliability. Last, given the findings of neuropathologic studies, we cannot exclude the blooming artifacts of the SWI sequence; thus, the exact depth of SS might have been overestimated.

\section{CONCLUSIONS}

In the present study, we found a high rate of widespread SS and ES in premature infants with GM-IVH. As hypothesized, the 
presence and extent of SS were closely and independently related to TVHV and GM-IVH grade. Notably, SS of the CN VII/VIII complex seems to be a very common complication of GM-IVH. Brain stem and cerebellar SS seem to be related to intraventricular hemorrhage, rather than to cerebellar hemorrhage. Although the deleterious effects of excessive iron on neuronal tissues are well-known, studies in premature infants with GM-IVH are limited and mostly retrospective. Prospective studies in preterm infants with GM-IVH are needed to clearly determine the clinical consequences of neonatal SS, especially relating to hearing impairment.

Disclosures: Garrett Smith—UNRELATED: Employment: University of Florida, Comments: I am an MD/PhD candidate at the University of Florida. I receive a regular stipend for this engagement.

\section{REFERENCES}

1. Kumar N, Cohen-Gadol AA, Wright RA. Superficial siderosis. Neurology 2006;66:1144-52 CrossRef Medline

2. Kumar N. Neuroimaging in superficial siderosis: an in-depth look. AJNR Am J Neuroradiol 2010;31:5-14 CrossRef Medline

3. Glasier CM, Garcia-Thomas GI, Allison JW. Superficial CNS siderosis in the newborn: MR diagnosis. Pediatr Radiol 1999;29:76-77 CrossRef Medline

4. Gomori JM, Grossman RI, Goldberg HI, et al. High-field spin-echo MR imaging of superficial and subependymal siderosis secondary to neonatal intraventricular hemorrhage. Neuroradiology 1987;29:339-42 CrossRef Medline

5. Yilmaz U, Meyer S, Gortner L, et al. Superficial siderosis after germinal matrix hemorrhage. AJNR Am J Neuroradiol 2016;37:2389-91 CrossRef Medline

6. Mittal S, Wu Z, Neelavalli J, et al. Susceptibility-weighted imaging: technical aspects and clinical applications, Part 2. AJNR Am J Neuroradiol 2009;30:232-52 CrossRef Medline

7. Tong KA, Ashwal S, Obenaus A, et al. Susceptibility-weighted MR imaging: a review of clinical applications in children. AJNR Am J Neuroradiol 2008;29:9-17 CrossRef Medline

8. Intrapiromkul J, Northington F, Huisman T, et al. Accuracy of head ultrasound for the detection of intracranial hemorrhage in preterm neonates: comparison with brain MRI and susceptibilityweighted imaging. J Neuroradiol 2013;40:81-88 CrossRef Medline

9. Papile LA, Burstein J, Burstein R, et al. Incidence and evolution of subependymal and intraventricular hemorrhage: a study of infants with birth weights less than 1,500 gm. J Pediatr 1978;92:529-34 CrossRef Medline

10. Tomayko MM, Reynolds CP. Determination of subcutaneous tumor size in athymic (nude) mice. Cancer Chemother Pharmacol 1989;24:148-54 CrossRef Medline
11. Willmore LJ, Hiramatsu M, Kochi H, et al. Formation of superoxide radicals after FeCI 3 injection into rat isocortex. Brain Res 1983;277:393-96 CrossRef Medline

12. Willmore LJ, Sypert GW, Munson JB. Recurrent seizures induced by cortical iron injection: a model of posttraumatic epilepsy. Ann Neurol 1978;4:329-36 CrossRef Medline

13. Koeppen AH, Michael SC, Li D, et al. The pathology of superficial siderosis of the central nervous system. Acta Neuropathol 2008;116:371-82 CrossRef Medline

14. Hughes JT, Oppenheimer DR. Superficial siderosis of the central nervous system: a report on nine cases with autopsy. Acta Neuropathol 1969;13:56-74 CrossRef Medline

15. Kellermier H, Wang G, Wiley C. Iron localization in superficial siderosis of the central nervous system. Neuropathology 2009;29:18795 CrossRef Medline

16. Koeppen AH, Barron KD. Superficial siderosis of the central nervous system. A histological, histochemical and chemical study. J Neuropathol Exp Neurol 1971;30:448-69 CrossRef Medline

17. Koeppen AH, Borke RC. Experimental superficial siderosis of the central nervous system. I: morphological observations. J Neuropathol Exp Neurol 1991;50:579-94 CrossRef Medline

18. Koeppen AH, Hurwitz CG, Dearborn RE, et al. Experimental superficial siderosis of the central nervous system: biochemical correlates. J Neurol Sci 1992;112:38-45 CrossRef Medline

19. Armstrong DL, Sauls CD, Goddard-Finegold J. Neuropathologic findings in short-term survivors of intraventricular hemorrhage. Am J Dis Child 1987;141:617 CrossRef Medline

20. Savman K, Nilsson UA, Blennow M, et al. Non-protein-bound iron is elevated in cerebrospinal fluid from preterm infants with posthemorrhagic ventricular dilatation. Pediatr Res 2001;49:208-12 CrossRef Medline

21. Back SA, Luo NL, Borenstein NS, et al. Late oligodendrocyte progenitors coincide with the developmental window of vulnerability for human perinatal white matter injury. J Neurosci 2001;21:130212 CrossRef Medline

22. Rakic P. Elusive radial glial cells: historical and evolutionary perspective. Glia 2003;43:19-32 CrossRef Medline

23. Fukumizu M, Takashima S, Becker LE. Glial reaction in periventricular areas of the brainstem in fetal and neonatal posthemorrhagic hydrocephalus and congenital hydrocephalus. Brain Dev 1996;18:40-45 CrossRef Medline

24. Nadol JB, Adams JC, O'Malley JT. Temporal bone histopathology in a case of sensorineural hearing loss caused by superficial siderosis of the central nervous system and treated by cochlear implantation. Otol Neurotol 2011;32:748-55 CrossRef Medline

25. da Silva LS, Ribeiro GE, Montovani JC, et al. The effect of peri-intraventricular hemorrhage on the auditory pathway of infants. Int J Pediatr Otorhinolaryngol 2018;112:24-26 CrossRef Medline 